\title{
Author Correction: The unpolarized macronova associated with the gravitational wave event GW 170817
}

S. Covino, K. Wiersema, Y. Z. Fan, K. Toma, A. B. Higgins, A. Melandri, P. D'Avanzo, C. G. Mundell, E. Palazzi, N. R. Tanvir, M. G. Bernardini, M. Branchesi, E. Brocato, S. Campana, S. di Serego Alighieri, D. Götz, J. P. U. Fynbo, W. Gao, A. Gomboc, B. Gompertz, J. Greiner, J. Hjorth, Z. P. Jin, L. Kaper, S. Klose, S. Kobayashi, D. Kopac, C. Kouveliotou, A. J. Levan, J. Mao, D. Malesani, E. Pian, A. Rossi, R. Salvaterra, R. L. C. Starling, I. Steele, G. Tagliaferri, E. Troja, A. J. van der Horst and R. A. M. J. Wijers

Correction to: Nature Astronomy https://doi.org/10.1038/s41550-017-0285-z; published online 16 October 2017.

In the version of this Letter originally published, in the third paragraph of the text Kyutoku et al. were not correctly cited and the sentence should have read: "As pointed out by Kyutoku et al. ${ }^{28}$, in the case of high optical depth to electron scattering $(\sim 1)$ and assuming spectral lines do not significantly depolarize the global emission, the linear polarization observed from the equatorial plane could be as high as a few per cent." Also, in the Author contributions section, the final sentence should have read: "C.G.M. contributed to the writing of the paper."

Published online: 30 October 2017

https://doi.org/10.1038/s41550-017-0319-6 